\title{
BMJ Open Exploring effects of presurgical weight loss among women with stage $0-$ II breast cancer: protocol for a randomised controlled feasibility trial
}

\author{
Yuko Tsuruta, ${ }^{1}$ Laura Q Rogers, ${ }^{1}$ Helen Krontiras, ${ }^{2}$ William E Grizzle, ${ }^{3}$ \\ Andrew D Frugé, ${ }^{1}$ Robert A Oster, ${ }^{4}$ Heidi R Umphrey, ${ }^{5}$ Lee W Jones, ${ }^{6}$ \\ Maria Azrad, ${ }^{1}$ Wendy Demark-Wahnefried $^{1}$
}

To cite: Tsuruta $Y$ Rogers LQ, Krontiras $\mathrm{H}$, et al. Exploring effects of presurgical weight loss among women with stage $0-$ II breast cancer: protocol for a randomised controlled feasibility trial. BMJ Open 2016:6:e012320. doi:10.1136/bmjopen-2016012320

- Prepublication history for this paper is available online. To view these files please visit the journal online (http://dx.doi.org/10.1136/ bmjopen-2016-012320).

Received 16 April 2016 Revised 1 July 2016 Accepted 12 August 2016

CrossMark

For numbered affiliations see end of article.

Correspondence to

Yuko Tsuruta;

tsuru@uab.edu

\section{ABSTRACT}

Introduction: Obesity is a known risk factor for postmenopausal breast cancer and is associated with poorer prognosis for premenopausal and postmenopausal patients; however, the aetiological mechanisms are unknown. Preclinical studies support weight loss via caloric restriction and increased physical activity as a possible cancer control strategy, though few clinical studies have been conducted. We undertook a feasibility trial among women recently diagnosed with stage 0-II breast cancer hypothesising that presurgical weight loss would be feasible, safe and result in favourable changes in tumour markers and circulating biomarkers.

Methods and analysis: A two-arm randomised controlled trial among 40 overweight or obese women, newly diagnosed with stage 0 -II breast cancer and scheduled for surgery was planned. The attention control arm received upper body progressive resistance training and diet counselling to correct deficiencies in nutrient intake; the experimental arm received the same plus counselling on caloric restriction and aerobic exercise to achieve a weight loss of $0.68-0.919 \mathrm{~kg} /$ week. In addition to achieving feasibility benchmarks (accruing and retaining at least $80 \%$ of participants, and observing no serious adverse effects attributable to the intervention), we will explore the potential impact of an acute state of negative energy balance on tumour proliferation rates (Ki-67), as well as other tumour markers, serum biomarkers, gene expression, microbiome profiles and other clinical outcomes (eg, quality of life). Outcomes for the 2 study arms are compared using mixed models repeated-measures analyses.

Ethics and dissemination: Ethics approval was received from the University of Alabama at Birmingham Institutional Review Board (Protocol number F130325009). Study findings will be disseminated through peer-reviewed publications. Given that this is one of the first studies to investigate the impact of negative energy balance directly on tumour biology in humans, larger trials will be pursued if results are favourable.

Trial registration number: NCT02224807; Pre-results.

\section{Strengths and limitations of this study}

- This is a novel presurgical weight-loss trial for women with stage 0 -II breast cancer that will explore the effect of an acute period of negative energy balance on tumour biology on a molecular level as well as circulating biomarkers within the host environment.

- This study is aimed at feasibility and therefore may lack the power to determine the impact of negative energy balance on tumour biology and relevant circulating biomarkers.

\section{INTRODUCTION}

Breast cancer is the second most common cancer among women in the USA after skin cancer. In 2016, an estimated 61000 new cases of ductal carcinoma in situ (DCIS) and 246660 new cases of invasive breast cancer will be diagnosed, with 40450 individuals dying of this disease. ${ }^{12}$ Many studies have shown that overweight and obesity are associated with an increased risk of postmenopausal breast cancer; most recently comprehensive meta-analysis has reported that the relative risk of postmenopausal breast cancer was 1.05 (95\% CI 1.03 to 1.07 ) per $2 \mathrm{~kg} / \mathrm{m}^{2}$ increase in body mass index (BMI). ${ }^{3}$ Moreover, obesity is linked to poorer prognosis, that is, increased disease-specific and overall mortality, among premenopausal and postmenopausal women. ${ }^{4}$ Currently, the exact mechanisms that link obesity to cancer progression are unknown, though it is surmised that complex interactions among multiple factors are involved. ${ }^{5-7}$ In breast cancer, various growth factors, inflammatory markers, adipokines and hormones, such as oestradiol and insulin, are likely to play roles and are influenced by weight status. ${ }^{8-10}$ It is speculated that the microbiome also may 
play a role in breast cancer related to systemic oestrogen levels and obesity. ${ }^{11}{ }^{12}$ Similarly, there are numerous observational studies linking insulin to breast cancer. Several weight loss intervention trials have shown that negative energy balance via caloric restriction and/or increased physical activity results in lower insulin levels. ${ }^{5-7} 13$ It has been proposed that weight loss has potential as a therapeutic strategy to slow disease progression, not only for women with DCIS, but also for those who have invasive disease. ${ }^{14-16}$ We aim to explore the feasibility of weight loss in this patient population, and describe the potential impact that weight loss invokes on tumour biology and the host environment, assessing effects on biomarkers and gene expression in the tumour and circulation using serum and peripheral blood mononuclear cells (PBMCs).

This research builds on our similarly designed phase II randomised controlled trial (RCT) in prostate cancer, in which we also use a presurgical model to explore whether we are able to affect tumour biology with brief exposure to a modified diet. ${ }^{17}$ While analyses of those results are currently underway, we observed significantly reduced tumour proliferation rates (Ki-67) in men with prostate cancer who received $30 \mathrm{~g}$ of ground flaxseed daily in another presurgical trial which also employed a 3-week intervention period. ${ }^{18}$ Secondary analysis showed that tumour levels of cytokines and angiogenic factors associated with the convergence of hormones, inflammation and energy-related factors (CHIEF) pathway, for example, vascular endothelial growth factor (VEGF), were also responsive to the brief period of dietary manipulation, as well as to weight status. ${ }^{8}{ }^{18}$ We postulate similar effects in early-stage breast cancer through biological and biobehavioural pathways in which weight loss may affect cancer progression.

\section{METHODS AND ANALYSIS}

Study design

Initially, the study was designed as a two-arm presurgical RCT among 40 overweight or obese postmenopausal women with DCIS (stage 0 breast cancer) in which overweight and obesity are hypothesised as key in the aetiology and progression of disease. However, due to slow accrual and because obesity is related to mortality regardless of stage and age, we expanded our eligibility criteria to include premenopausal and peri-menopausal women, as well as those with stage I and II breast cancer. ${ }^{4}$ Consenting patients are randomised to one of the two study arms after completion of their baseline assessment. Attention control and experimental arms receive diet counselling during the study period to correct nutritional deficiencies with food sources, as well as instruction on progressive resistance training (PRT) of the upper body using Therabands (Hygenic Corporation, Akron, Ohio, USA). The experimental arm receives PRT plus mentored aerobic exercise and diet counselling for caloric restriction to promote a weight loss of up to $0.91 \mathrm{~kg} /$ week (figure 1). Participants in each of the study arms are contacted at least twice weekly by study staff. All participants undergo follow-up assessment immediately prior to surgery.

\section{Outcomes}

While the primary outcomes relate to feasibility, that is, whether the study is able to accrue and retain at least $80 \%$ of the sample and result in no serious adverse events attributable to the intervention, a key biological outcome of interest is tumour proliferation rates (Ki-67) in breast tumours and tissue between the experimental and attention control arms. Based on the results of our prior diet and exercise trials, we speculate that in comparison to the attention control arm with stable weight, the experimental arm will lose weight and exhibit decreases in Ki-67, though we acknowledge that such analyses are exploratory and may be insufficiently powered. While our accrual has been slower than anticipated, thus far and with more than half of our sample enrolled, we have observed excellent rates of retention and no serious adverse events.

Secondary outcomes are to explore improvements in other biomarkers. These changes are studied in relation to the following end points: (1) adiposity measures, that is, body weight, waist circumference (WC) and per cent body fat from dual-energy X-ray absorptiometry (DXA); (2) energy intake and nutrient density of the diet; (3) time, frequency, and intensity of physical activity via selfreport and accelerometry; (4) cardiorespiratory fitness; (5) tumour markers and phosphoro-proteins on the CHIEF pathway in tumour; (6) gene expression profiles in tumour and PBMCs; (7) serum levels of insulin, leptin, estradiol, VEGF, tumour necrosis factor $\alpha$ (TNF $\alpha$ ), and sex hormone-binding globulin (SHBG); (8) changes in the salivary and faecal microbiome; and (9) quality of life (QoL).

\section{Recruitment/consent}

Cases are recruited from the University of Alabama at Birmingham (UAB) Kirklin Interdisciplinary Breast Health Clinic (Birmingham, Alabama, USA). A thorough explanation of the study and printed materials are provided to all patients who meet general screening criteria, that is, overweight or obese (BMI of $25-60 \mathrm{~kg} / \mathrm{m}^{2}$ ) with histopathologically confirmed stage $0-$ II breast cancer who will receive surgical treatment. Data on age, race, $\mathrm{BMI}$ and mileage from residence to $\mathrm{UAB}$ are $\mathrm{col}^{-}$ lected on all patients approached; these data are de-identified and stored with the stated reason for refusal or reasons for ineligibility. At trial completion, we will analyse data to discern if refusers and ineligibles differ from enrolees on demographic factors since such data are vital to assess generalisability of findings.

\section{Eligibility and exclusion}

This study accrues overweight or obese women with stage 0-II breast cancer who: (1) elect surgery with 
Overweight/Obese women with stage 0 -II breast cancer electing surgery

Eligibility assessed via patient interview, medical history, and biopsy pathology reports; minimum 3 week intervention period

Written informed consent

\section{Baseline Assessment}

Anthropometric measures, dietary intake, physical activity (selfreport/accelerometry), cardiorespiratory fitness, biomarkers, quality of life, demographics, and comorbidities

Those who consent to re-biopsy will be biopsied under ultrasound and tissue will be flash frozen.

Randomization stratified by BMI $25-29.9$ or $30-60$

\begin{tabular}{|c|c|}
\hline $\begin{array}{c}\text { Attention Control Arm } \\
(\mathrm{n}=20)\end{array}$ & $\begin{array}{c}\text { Experimental Arm [weight loss] } \\
(\mathrm{n}=20)\end{array}$ \\
\hline $\begin{array}{c}\text { Upper body PRT }+ \\
\text { general diet counseling }\end{array}$ & $\begin{array}{c}\text { Upper body PRT }+ \\
\text { mentored aerobic exercise }+ \\
\text { diet counseling for calorie restriction }\end{array}$ \\
\hline
\end{tabular}

\section{$\downarrow$ \\ Follow-up Assessment \\ (prior to surgery)}

Identical measures taken at the baseline are repeated except height and demographics

Fresh frozen and fixed tumor obtained at surgery.

Figure 1 Study schema. BMI, body mass index; PRT, progressive resistance training.

more than 3 weeks lag-time between the start of the intervention and their scheduled surgery; (2) have no pre-existing medical conditions that preclude adherence to unsupervised exercise-we screen potential participants with the Physical Activity Readiness Questionnaire (PAR-Q), ${ }^{19}$ and obtain physician clearance for any conditions affirmed by patients, including resting blood pressures $>99$ diastolic or $>159$ systolic or cardiac abnormality; (3) have no current medical condition that affects weight status, such as Cushing's syndrome or untreated hypothyroidism; (4) have no additional active malignancy; (5) are English speaking/reading since all survey instruments are not validated in other languages; (6) are not currently enrolled in a weight loss programme; and (7) are willing to be assigned to either study arm and adhere to the protocol. Written informed consent is obtained from all interested and eligible women.

\section{Baseline assessment}

Study measures are outlined in table 1. Data regarding scheduled surgery, demographics, medical history, medication-use and comorbidities (Older American Resources and Services (OARS) comorbidity scale) are collected and logged..$^{20-22}$ QoL is measured, given the potential of weight loss to improve emotional well-being and overall health. Of the many instruments to measure QoL, the Functional Assessment of Cancer Therapy for Breast Cancer (FACT-B) was selected as it assesses weight concerns and has been validated in newly diagnosed 
Table 1 Schedule for measurement, testing and assessment at baseline and follow-up visits

\begin{tabular}{|c|c|c|}
\hline Measures & Baseline & Follow-up \\
\hline \multicolumn{3}{|l|}{ Anthropometric measures } \\
\hline Weight & $\mathrm{X}$ & $\mathrm{X}$ \\
\hline Height & $\mathrm{X}$ & \\
\hline BMI $\left(\mathrm{kg} / \mathrm{m}^{2}\right)$ & $\mathrm{X}$ & $\mathrm{x}$ \\
\hline Waist circumference & $\mathrm{X}$ & $\mathrm{X}$ \\
\hline Body composition (DXA) & $\mathrm{X}$ & $\mathrm{X}$ \\
\hline \multicolumn{3}{|l|}{ Components of energy balance (antecedents to change in BMI) } \\
\hline Dietary intake (1-weekday and 1-weekend day) by a registered dietitian & $\mathrm{X}$ & $\mathrm{X}$ \\
\hline Godin leisure time physical activity questionnaire & $\mathrm{X}$ & $\mathrm{X}$ \\
\hline Physical activity data captured via accelerometry (3-day period) & $\mathrm{X}$ & $\mathrm{X}$ \\
\hline Cardiorespiratory fitness $\left(\mathrm{VO}_{2}\right.$ submax $)$ & $\mathrm{X}$ & $\mathrm{X}$ \\
\hline \multicolumn{3}{|l|}{ Tumour markers } \\
\hline $\begin{array}{l}\text { Ki-67, I-R, VEGF, TNF } \alpha, N F \kappa B, 4 E-B P 1 \text {, activated caspase-3 and } p 16 \text { in paraffin-embedded } \\
\text { tumours }\end{array}$ & $\mathrm{X}$ & $\mathrm{X}$ \\
\hline 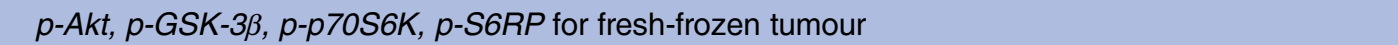 & $X^{*}$ & $\mathrm{X}$ \\
\hline \multicolumn{3}{|l|}{ Gene expression using multi array } \\
\hline Insulin (I-R), leptin (LEPR), VEGF (VEGFR) and TNF (FAS) in tumours & $\mathrm{X}$ & $\mathrm{X}$ \\
\hline Insulin (I-R), leptin (LEPR), VEGF (VEGFR), TNF (FAS) and apoptosis regulator (Bcl-2) in PBMCs & $\mathrm{X}$ & $\mathrm{x}$ \\
\hline \multicolumn{3}{|l|}{ Serum biomarkers } \\
\hline Insulin, leptin, estradiol, VEGF, TNF $\alpha$, SHBG & $\mathrm{X}$ & $\mathrm{x}$ \\
\hline \multicolumn{3}{|l|}{ Microbiome profiles } \\
\hline Saliva & $\mathrm{X}$ & $\mathrm{X}$ \\
\hline Faeces & $X$ & $\mathrm{X}$ \\
\hline \multicolumn{3}{|l|}{ Quality of life } \\
\hline FACT-B (questionnaire) & $\mathrm{X}$ & $x$ \\
\hline \multicolumn{3}{|l|}{ Demographics/comorbidities } \\
\hline Demographics - race, age, educational level & $X$ & \\
\hline Medical history and medications & $\mathrm{X}$ & $\mathrm{X}$ \\
\hline OARS comorbidity scale & $\mathrm{X}$ & $\mathrm{X}$ \\
\hline 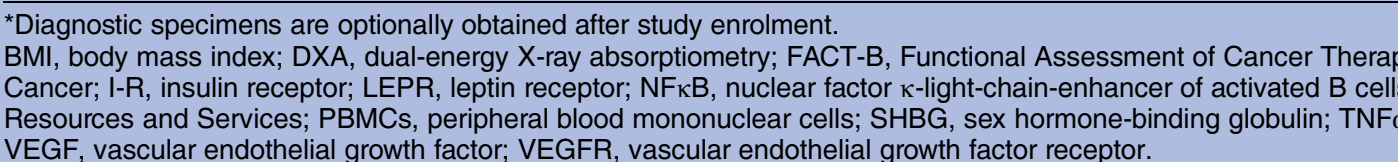 & $\begin{array}{l}\text { Questionnai } \\
\text { OARS, Olde } \\
\text { tumour necr }\end{array}$ & $\begin{array}{l}\text { re for Breast } \\
\text { os American } \\
\text { osis factor } \alpha \text {; }\end{array}$ \\
\hline
\end{tabular}

patients with breast cancer. ${ }^{23}$ Self-reported physical activity is collected using the Godin Leisure Time Physical Activity Questionnaire. ${ }^{24}$ A programmed accelerometer (wGT3X: Actigraph, LLC, Pensacola, Florida, USA), worn at the hip during waking hours ( 06:00 to 22:00), is used to capture objective activity data. While a 7-day collection period is ideal for accelerometry, the brevity of the presurgical period constrained our collection to 3 days. ${ }^{25}$ Epoch length during initialisation of the accelerometer is set to $60 \mathrm{~s}$, non-wear time is defined as 60 min of no activity and a valid day is defined as at least 10 hours of valid wear time. Moderate physical activity is defined as 1952-5724 counts/min, vigorous physical activity as $5725-9498$ counts/min and very vigorous as $>9498$ counts $/ \mathrm{min} .{ }^{26}$ Two dietary recalls ( 1 weekday and 1 weekend day) are taken at each time point using a multiple-pass method and the Nutrition Data System for Research (NDSR 2014, Minneapolis, Minnesota, USA) for dietary analysis.

$\mathrm{VO}_{2}$ SubMax testing (Trackmaster Treadmills Model TMX425CP, Full Vision., Newton, Kansas, USA) is performed per the guidelines of the American College of
Sports Medicine (ACSM) and using the Naughton protocol. Fitness is determined by estimating the oxygen cost of walking at the treadmill incline and speed achieved at $85 \%$ of predicted maximal heart rate (MHR) using published regression equations and expressed in $\mathrm{mL} / \mathrm{kg} /$ min. $^{27} 28$

Anthropometric measures of weight, height and WC are performed using protocols defined by the Anthropometric Standardisation Manual, with measures taken to the nearest tenth of a kilogram or centimetre. ${ }^{29}$ BMI is calculated using the Quetelet equation of $\mathrm{kg} / \mathrm{m}^{2}$. Body composition is assessed using DXA (Lunar iDXA, GE Healthcare, Waukeha, Wisconsin, USA) on a densitometer which is calibrated at least daily using an anthropomorphic phantom.

Fasting blood $(20 \mathrm{~mL})$ is collected and configured into sera, plasma and buffy coat. Half of the buffy coat is stored in RNAlater (Thermo Fisher Scientific, Grand Island, New York, USA) and the remaining buffy coat is stored as is. To assess any changes in the microbiome, $3 \mathrm{~mL}$ of saliva is collected in a test tube and faecal samples are collected using a sterile wipe. Recent 
antibiotic intake is self-reported on sample collection. All samples are stored at $-80^{\circ} \mathrm{C}$ until batch analysis.

If women consent to an additional biopsy, biopsies are performed with a $14 \mathrm{G}$ needle under ultrasound guidance and tissue is fresh frozen. The optional collection of fresh tissue at baseline is later compared with postintervention samples obtained at the time of surgery. All pathology reports of biopsies are reviewed for breast cancer subtype, and biopsy tissues are accessed for gene expression and immunohistochemistry. Paraffinembedded tissues are available at baseline for all participants. Given the brevity of the presurgical period, these assessments are scheduled within 1-week of diagnosis.

\section{Randomisation}

Given that this was a feasibility trial with power calculated to detect differences in weight change between study arms, we stratified on the primary factor that would differentiate future weight loss, that is, current BMI status. ${ }^{17}$ BMI groups of 25.5-29.9 and 30.0-60.0, and with block sizes of 4 , is used for this trial. At the initiation of the trial, randomisation sequences were generated by the study statistician (RAO) and assignments were placed in sealed envelopes. As participants complete their baseline assessments, their envelope is opened in the order it was generated and their assignment is recorded. Assignments are re-verified with the statistician to assure fidelity with the randomisation process.

\section{Attention control intervention}

An attention control arm is implemented to enhance interest in the trial and improve study accrual and retention. Given concern regarding lymphoedema, we provide PRT as a means of 'pre-habilitation' and strengthening the arm prior to surgery. ${ }^{30}{ }^{31}$ An ACSM-certified Cancer Exercise Trainer (CET) provides guidance on three resistance band exercises that target the triceps, biceps and deltoids. ${ }^{32}$ In addition, participants receive dietary counselling by a registered dietitian (RD) to correct nutrient deficiencies (with food sources rather than supplements) that are detected by the NDSR analysis of their 2-day dietary recalls conducted at baseline. ${ }^{33}$

\section{Experimental intervention}

The same PRT protocol and dietary guidance described above are offered as part of the experimental intervention. Experimental arm participants also receive additional intensive counselling to achieve a weight loss of $0.68-0.919 \mathrm{~kg} /$ week. We estimate energy needs using the Mifflin-St. Jeor equation and a multiplier which assumes sedentary behaviour, that is, $1.2(10 \times($ body weight in $\mathrm{kg})$ +6.25 (height in cm) - 5(age)) and then subtract 750$1000 \mathrm{kcal} /$ day. ${ }^{34}$ Participants are trained on how to achieve this caloric deficit through dietary caloric restriction and increased physical activity. Participants are provided a choice of either counting their calories, following a prescribed meal plan or using a 'Choose Your Foods' exchange list system endorsed by the
American Diabetes Association and the Academy of Nutrition and Dietetics. ${ }^{35} 36$ A healthy, nutritionally adequate diet that meets the Dietary Reference Intakes, with food choice patterns consistent with American Cancer Society and World Cancer Research Fund/ American Institute of Cancer Research guidelines is recommended. ${ }^{37-40}$ Guidance is provided regarding portion control, dietary restraint and replacing nutrient poor and high energy foods with nutrient rich and low energy foods.

An exercise programme is tailored taking into account energy expenditure for various activities; physical activity-associated expenditures of $200-400 \mathrm{kcal} /$ day serves as a goal. Aerobic training of large leg muscles is emphasised to achieve a greater energy deficit, and ramping of intensity and volume over time is pursued per ACSM guidelines. ${ }^{41}$ Participants train once to twice weekly under the supervision of the CET, and are encouraged to exercise at home using a heart rate monitor to assure fidelity to the training programme. Onsite weighing occurs at the beginning of each supervised training session.

Social cognitive theory (SCT) is used as the behavioural framework to guide the intervention. ${ }^{42}$ Self-monitoring is a key concept of SCT, and participants are provided with a scale, pedometers or Fitbits, and asked to log their body weight, foods eaten along with accompanying calorie or exchange list values and physical activity behaviours daily. The CET and RD interact with patients at least twice weekly and begin by reviewing current intake and activity levels, adjusting intake, fielding questions, providing support and problem-solving to overcome barriers, and setting goals for the upcoming week.

\section{Follow-up assessment}

On the day of or the day prior to surgery, a follow-up appointment is scheduled. At this time, measures taken at the baseline assessment are repeated, except for height and the demographic survey. At surgery, freshfrozen tumour tissue and adjacent adipose tissue is obtained. Later, paraffin-embedded tissue is collected from the Department of Pathology at UAB.

\section{Tissue processing}

All tumour blocks are reviewed by a pathologist at the Tissue Collection and Banking Facility at UAB to confirm atypical lesions. Immunostaining for Ki-67, insulin receptor (I-R), VEGF, TNF $\alpha$, nuclear factor $\kappa$-light-chain-enhancer of activated B cells (NFKB), 4E-BP1, activated caspase-3, and p16 is then performed. Whole tissue lysates are extracted from fresh-frozen tumour to determine levels of phosphorylated Akt, GSK-3 $\beta$, p70S6K and S6RP (phosphoproteins on the CHIEF pathway) in the Physiology and Metabolism core at UAB.

\section{Serum processing}

Serum assays for insulin, leptin, VEGF, TNF $\alpha$ and SHBG are performed in the Physiology and Metabolism core at 
UAB. Insulin and SHBG are assayed by immunofluorescence on a TOSOH AIA-600 II Automated Immunoassay Analyzer (TOSOH Bioscience, South San Francisco, California, USA); minimum sensitivity of $0.5 \mu \mathrm{U} / \mathrm{mL}$ for insulin and $0.104 \mathrm{nmol} / \mathrm{L}$ for SHBG, intra-assay coefficients of variance $(\mathrm{CV})$ of $1.49 \%$ for insulin and $1.61 \%$ for SHBG, and interassay CV of $4.42 \%$ for insulin and $5.60 \%$ for SHBG. Leptin is measured using a Human Leptin RIA kit (EMD Millipore, Billerica, Massachusetts, USA); minimum sensitivity of $0.92 \mathrm{ng} / \mathrm{mL}$, intra-assay $\mathrm{CV}$ of $6.61 \%$ and interassay CV of $7.13 \%$. VEGF and $\mathrm{TNF} \alpha$ are measured using a multiplex proinflammatory assay on the SECTOR imager 2400 (Meso Scale Diagnostics, Rockville, Maryland, USA); minimum sensitivity of $0.92 \mathrm{pg} / \mathrm{mL}$ for VEGF and $0.11 \mathrm{pg} / \mathrm{mL}$ for TNF $\alpha$, intra-assay CV of $8.63 \%$ for VEGF and $4.57 \%$ for $\mathrm{TNF} \alpha$, and interassay $\mathrm{CV}$ of $14.20 \%$ for VEGF and $10.90 \%$ for TNF $\alpha$.

Serum assays for estradiol are performed at the Quest Diagnostics Nichols Institute (San Juan Capistrano, California, USA). The Quest Diagnostics Nichols Institute performs oestrogen extraction and analysis using mass spectrometry which is important in postmenopausal women who often have minimal levels of oestrogen.

\section{Gene expression}

Gene expression analyses are performed on RNA from microdissected tumour specimens and PBMCs that are isolated from the buffy coat stored in RNAlater. Total RNA is extracted from the samples using a Trizol reagent (Invitrogen, Carlsbad, California, USA) and RNeasy Mini Kit (QIAGEN, Valencia, California, USA) according to the manufacturer's instructions. The concentration and purity of total RNA is determined using a 2100 Bioanalyzer (Agilent Technologies, Santa Clara, California, USA). At the UAB Comprehensive Cancer Center Shared Genomics Resource, samples are purified to cDNA. After conversion to biotin-labelled cRNA, they are hybridised to a HumanHT-12 v4 Expression BeadChip (Illumina, San Diego, California, USA) and stained with strepavidin-Cy3 for visualisation. Though select genes have been identified specifically on the CHIEF pathway, the HumanHT-12 v4 Expression BeadChip contains sequences representing 47231 curated and putative genes and expressed sequence tags. Quality standards for hybridisation, labelling, staining, background signal and basal level of housekeeping gene expression for each chip is verified on all assays. After scanning the probe array, the resulting image is analysed using GenomeStudio software (Illumina). Gene lists are created by exporting the data from GenomeStudio into GeneSpring (V.11.5.1, Agilent Technologies). The data inside GeneSpring are first normalised using the Robust Multiarray (RMA) method and background is subtracted from the median of all samples. Samples are then filtered based on the flags present and marginal, thereby removing absent flags.

\section{Gut microbiome}

At each time point, participants collect faecal and saliva samples in order to explore if changes in physical activity and dietary intake affect the microbiome. Faecal samples are collected after a bowel movement using a sterile wipe which is then placed in a plastic bag and immediately frozen. Saliva samples are collected in a $50 \mathrm{~mL}$ Falcon tube prior to brushing teeth on the assessment day. Both sets of specimens are stored at $-80^{\circ} \mathrm{C}$ and delivered to the Microbiome/Bioinformatic/ Gnotobiotic Animal Core at UAB where microbiome analysis is conducted.

Microbiome analysis targeting the $\mathrm{V} 4$ region of the 16S rRNA gene is performed using an Illumina MiSeq and employing similar quality assurance methods as described previously. ${ }^{7} 13 \quad{ }^{17}$ Analyses of the microbiome samples are performed using the Quantitative Insight into Microbial Ecology (QIIME) suite, V.1.7 and a QIIME wrapper (QWRAP) as described previously. ${ }^{43-45}$

\section{Power and sample size calculations}

Data from our previous UAB weight loss programmes indicate a mean weight loss over 3-4 weeks of 2.82 $\pm 1.57 \mathrm{~kg}$, which was used to estimate power. Initially, 40 participants with 20 per arm were planned, and a drop-out rate of $20 \%$ was assumed, that is, 16 per arm. Sample sizes of $14-16$ per arm yield $>90 \%$ power to detect a difference in the means of $2.82 \mathrm{~kg}$, assuming a two-group, two-sided t-test and a significance level of $5 \%$.

\section{Statistical analysis}

Given the exploratory nature of this work, our analysis will rely heavily on descriptive statistics, such as means, SDs, frequencies and proportions, which will be calculated for each study arm, and explored within subgroups of interest, that is, patients of differing cancer stage, and of premenopausal and postmenopausal status. Comparisons of baseline characteristics between enrolled participants and potential participants who refused to participate, as well as between participants who completed the study and those dropped out, are compared using the two-group t-test for continuous variables and the Pearson $\chi^{2}$ test for categorical variables. Biomarkers and anthropometric change scores are also compared between the groups using the two-group t-test. Overall changes in measures from baseline to postintervention may be examined using the paired t-test. Adverse events are summed as frequencies and are compared between the two groups using the Pearson $\chi^{2}$ test. The primary method of analysis will be mixed models repeated-measures analyses, such as repeated-measures analysis of covariance. When a model term is statistically significant, the Tukey-Kramer multiple comparisons test will be used to determine which specific pairs of means are significantly different. This method allows us to compare changes over time (within-group changes) and 
differences between groups simultaneously. These models include terms for group (study arm), time (baseline, follow-up) and group $\times$ time, as well as terms for interactions that are of scientific interest. Variables such as select demographic (eg, race and menopausal status) and tumour factors (eg, cancer stage) are accounted for in these analyses.

Relationships between serum and tissue markers and adiposity are examined using the Pearson correlation analysis. Distributions of continuous variables are examined using stem-and-leaf plots, normal probability plots and the Kolmogorov-Smirnov test. Variables that are determined to deviate greatly from a normal distribution are transformed prior to analysis or are analysed using non-parametric tests such as the Wilcoxon rank-sum and signed-rank tests. Statistical tests are two-sided and are performed using a 5\% significance level. SAS software (V.9.4; Cary, North Carolina, USA) is used to conduct the statistical analyses.

\section{Trial status}

This trial opened for recruitment 21 August 2014, with recruitment expected to be completed by August 2016 . At the time of submission of this study protocol, 85 potentially eligible patients have been approached, 57 were found to be ineligible, 28 patients were enrolled and 26 patients have completed the study. One patient was excluded before the follow-up assessment due to findings of advanced cancer after the baseline assessment. Currently, our rate of drop-out is $3.5 \%$ (far lower than the $20 \%$ forecasted). One of the greatest barriers to recruitment has been the required 3 week lagtime between baseline assessment and surgery, as many prospective participants elect to have surgery as soon as possible. Other reasons for ineligibility are patient's overwhelming distress resulting from their cancer diagnosis and expressed concern that they 'just can't take on anything else', job/time conflicts and other responsibilities such as a caregiver role for family members. Currently, none of the enrolled patients have volunteered for an additional biopsy at baseline assessment; while this will preclude our ability to compare biomarkers in fresh-frozen tissue, we still will be able to conduct all assessments planned on paraffin-embedded samples.

\section{DISSEMINATION}

As such, all adverse events are recorded and classified as either serious or non-serious. Serious adverse events include conditions that are fatal or life threatening, permanently disabling or which require prolonged hospitalisation. All other adverse events are considered non-serious. To date, no serious adverse events and nonserious events have occurred. The results of the study will be published in a peer-reviewed scientific journal after study completion.

\section{DISCUSSION}

This is the first trial to track the effects of a presurgical weight loss intervention in women with early-stage breast cancer. Our study is unique because it uses a presurgical model which allows the opportunity to explore the impact of acute negative energy balance with weight loss directly on the tumour and at a molecular level, as well as systemically assessing select markers, gene expression, tumour proliferation and apoptosis, and QoL (figure 2).

The CHIEF model proposed by Slattery and Fitzpatrick ${ }^{8}$ provides a unifying framework that incorporates much of the energy, hormonal and inflammatory responses associated with energy balance. Our study will explore effects on metabolic markers within the CHIEF pathway as mediators by which negative energy balance affects tumour growth. Downstream of the CHIEF pathway are the PI3K and the mTOR signalling pathways which are currently being evaluated for drug

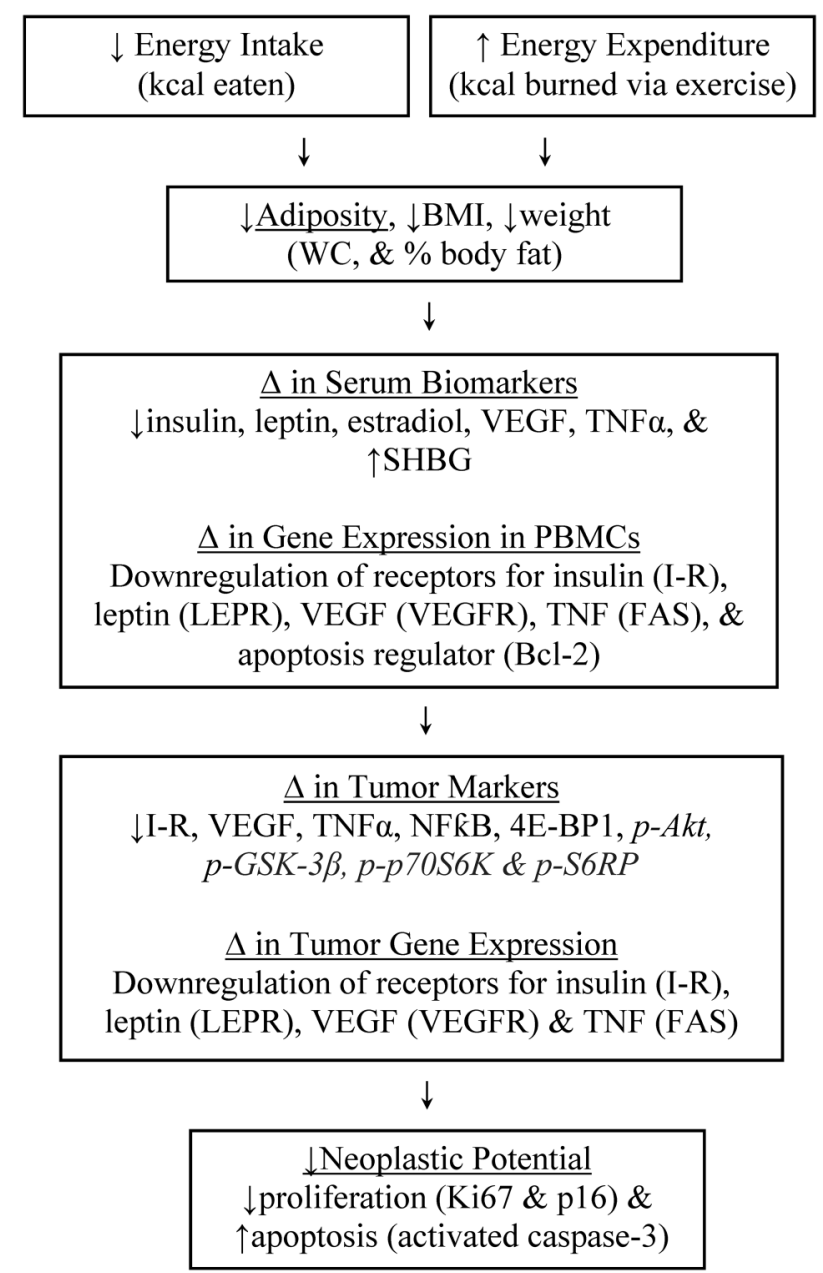

Figure 2 Logic model linking negative energy balance to increased tumour suppression. BMI, body mass index; I-R, insulin receptor; LEPR, leptin receptor; $N F_{\kappa} B$, nuclear factor $\kappa$-light-chain-enhancer of activated $B$ cells; PBMCs, peripheral blood mononuclear cells; SHBG, sex hormone-binding globulin; TNF $\alpha$, tumour necrosis factor $\alpha$; VEGF, vascular endothelial growth factor; VEGFR, vascular endothelial growth factor receptor; WC, waist circumference. 
development and are also affected by caloric restriction and increased physical activity. ${ }^{46}$

Our primary study limitations include a lack of statistical power to determine anything more than the feasibility of weight loss. Moreover, the sheer number of assessments in combination with a small sample size may increase the type 1 error, and result in inconclusive findings. However, it must be borne-in-mind that is a feasibility trial and one in which the exploration of other end points is strictly for the purposes of hypothesis generating (not testing). Another limitation is the substantial trade-offs inherent when a study is dependent on and integrated within a pre-surgical clinical care timeline. While it would be ideal to delay surgery more than a minimum of 3 weeks, in order to allow for longer accelerometry collection periods as well as longer intervention periods, further delay is seldom acceptable within the US healthcare system which schedules women for surgery on demand. Although the study period is brief, we will explore differences in tumour proliferation rates and cancer-related biomarkers within the span of 3 weeks as experienced in a similar study among patients with prostate cancer. ${ }^{18}$

In summary, this study builds on our prior work and extant clinical practice, utilises an appropriate and accessible patient population and is designed to gather data that will begin to improve our understanding of the effect of weight loss on breast cancer biology and related biomarkers and outcomes. Since weight loss is apt to be pursued as complementary care in the clinical setting, this study is further strengthened by its real-world approach. Indeed, we expect to uncover exciting findings that could ultimately influence the standard of care for treatment of early-stage breast cancer.

\section{Author affiliations}

${ }^{1}$ Department of Nutrition Sciences, University of Alabama at Birmingham, Birmingham, Alabama, USA

${ }^{2}$ Department of Surgery, University of Alabama at Birmingham, Birmingham, Alabama, USA

${ }^{3}$ Department of Pathology, University of Alabama at Birmingham, Birmingham, Alabama, USA

${ }^{4}$ Department of Medicine, University of Alabama at Birmingham, Birmingham, Alabama, USA

${ }^{5}$ Department of Radiology, University of Alabama at Birmingham, Birmingham, Alabama, USA

${ }^{6}$ Department of Medicine, Memorial Sloan Kettering Cancer Center, New York, New York, USA

Acknowledgements The authors thank the efforts of Dr Kimberly Keene, Dr Catherine Parker, Dr Jennifer De Los Santos, Dr Drexell Boggs, Dr Marshall Urist, Dr Carla Falkson, Dr Andres Forero, Dr Kirby Bland, Dr Chris Vaklavas, Dr Erica Stringer-Reasor and Dr Markus Bredel, Alma del Grosso, RN, Kathleen Romundstad, RN, Sara Mansfield, MS, ACSM Certified Exercise Physiologist (CET), Alexandra Yates, MS, ACSM Certified Exercise Physiologist (CET), Britney Blackstock, Heather Hunter, MS, Madeline Morgan, and Lora Roberson. Finally, the authors acknowledge the efforts of their study participants without whom this research would not have been possible.

Contributors YT contributes to study management, analysis, writing and review of manuscript. LQR contributes to study design, intervention assessment and manuscript review. HK contributes to participant recruitment. WEG contributes to study design and histology assessment.
ADF contributes to study management, intervention delivery and review of manuscript. RAO contributes to study design, statistical analysis and manuscript review. LWJ contributes to conception and study design. MA contributes to study management. WD-W, chief investigator, contributes to conception, study design, study management, analysis, writing and review of manuscript.

Funding This work was supported by the National Cancer Institute (1R21CA178359-01A1). LWJ is supported by research grants from the National Cancer Institute, AKTIV Against Cancer and the Memorial Sloan Kettering Cancer Center Support Grant/Core Grant (P30 CA008748).

Competing interests None declared.

Patient consent Obtained.

Ethics approval This study is a phase II trial which has been approved and monitored by the University of Alabama at Birmingham Institutional Review Board for Human Use (Protocol number F130325009).

Provenance and peer review Not commissioned; externally peer reviewed.

Open Access This is an Open Access article distributed in accordance with the Creative Commons Attribution Non Commercial (CC BY-NC 4.0) license, which permits others to distribute, remix, adapt, build upon this work noncommercially, and license their derivative works on different terms, provided the original work is properly cited and the use is non-commercial. See: http:// creativecommons.org/licenses/by-nc/4.0/

\section{REFERENCES}

1. Siegel RL, Miller KD, Jemal A. Cancer statistics, 2016. CA Cancer $J$ Clin 2016;66:7-30.

2. Susan G K. Breast cancer statistics. Secondary breast cancer statistics. 2016. http://ww5.komen.org/BreastCancer/Statistics.html (accessed 28 Feb 2016).

3. Eheman C, Henley SJ, Ballard-Barbash R, et al. Annual Report to the Nation on the status of cancer, 1975-2008, featuring cancers associated with excess weight and lack of sufficient physical activity. Cancer 2012;118:2338-66.

4. Protani M, Coory M, Martin JH. Effect of obesity on survival of women with breast cancer: systematic review and meta-analysis. Breast Cancer Res Treat 2010;123:627-35.

5. Hursting SD, Berger NA. Energy balance, host-related factors, and cancer progression. J Clin Oncol 2010;28:4058-65.

6. Demark-Wahnefried W, Platz EA, Ligibel JA, et al. The role of obesity in cancer survival and recurrence. Cancer Epidemiol Biomarkers Prev 2012;21:1244-59.

7. McTiernan A, Irwin M, Vongruenigen V. Weight, physical activity, diet, and prognosis in breast and gynecologic cancers. J Clin Oncol 2010;28:4074-80.

8. Slattery ML, Fitzpatrick FA. Convergence of hormones, inflammation, and energy-related factors: a novel pathway of cancer etiology. Cancer Prev Res (Phila) 2009;2:922-30.

9. Xuan C, Shamonki JM, Chung A, et al. Microbial dysbiosis is associated with human breast cancer. PLOS ONE 2014;9:e83744.

10. Gunter MJ, Wang T, Cushman M, et al. Circulating adipokines and inflammatory markers and postmenopausal breast cancer risk. $J$ Natl Cancer Inst 2015;107:pii:djv169.

11. Goedert JJ, Jones G, Hua X, et al. Investigation of the association between the fecal microbiota and breast cancer in postmenopausal women: a population-based case-control pilot study. J Natl Cancer Inst 2015;107:pii:djv147.

12. Bradlow HL. Obesity and the gut microbiome: pathophysiological aspects. Horm Mol Biol Clin Investig 2014;17:53-61.

13. Ballard-Barbash R, Hunsberger S, Alciati MH, et al. Physical activity, weight control, and breast cancer risk and survival: clinical trial rationale and design considerations. J Natl Cancer Inst 2009;101:630-43.

14. Calle EE, Rodriguez C, Walker-Thurmond K, et al. Overweight, obesity, and mortality from cancer in a prospectively studied cohort of U.S. adults. N Engl J Med 2003;348:1625-38.

15. Neuhouser ML, Aragaki AK, Prentice RL, et al. Overweight, obesity, and postmenopausal invasive breast cancer risk: a secondary analysis of the women's health initiative randomized clinical trials. JAMA Oncol 2015;1:611-21.

16. Morimoto LM, White E, Chen Z, et al. Obesity, body size, and risk of postmenopausal breast cancer: the Women's Health Initiative (United States). Cancer Causes Control 2002;13:741-51. 
17. Demark-Wahnefried W, Nix JW, Hunter GR, et al. Feasibility outcomes of a presurgical randomized controlled trial exploring the impact of caloric restriction and increased physical activity versus a wait-list control on tumor characteristics and circulating biomarkers in men electing prostatectomy for prostate cancer. BMC Cancer 2016;16:61.

18. Demark-Wahnefried W, George SL, Switzer BR, et al. Overcoming challenges in designing and implementing a phase II randomized controlled trial using a presurgical model to test a dietary intervention in prostate cancer. Clin Trials 2008;5:262-72.

19. Shephard RJ. PAR-Q, Canadian Home Fitness Test and exercise screening alternatives. Sports Med 1988;5:185-95.

20. Fillenbaum GG, Smyer MA. The development, validity, and reliability of the OARS multidimensional functional assessment questionnaire. $J$ Gerontol 1981;36:428-34.

21. George LK, Fillenbaum GG. OARS methodology. A decade of experience in geriatric assessment. J Am Geriatr Soc 1985;33:607-15.

22. Moore JT, Fillenbaum GG. Change in functional disability of geriatric patients in a family medicine program: implications for patient care. $J$ Fam Pract 1981;12:59-66.

23. Cella DF, Tulsky DS, Gray G, et al. The functional assessment of cancer therapy scale: development and validation of the general measure. J Clin Oncol 1993;11:570-9.

24. Amireault S, Godin G, Lacombe J, et al. The use of the GodinShephard leisure-time physical activity questionnaire in oncology research: a systematic review. BMC Med Res Methodol 2015;15:60.

25. Cain KL, Sallis JF, Conway TL, et al. Using accelerometers in youth physical activity studies: a review of methods. J Phys Act Health 2013;10:437-50.

26. Freedson PS, Melanson E, Sirard J. Calibration of the computer science and applications, Inc. accelerometer. Med Sci Sports Exerc 1998;30:777-81.

27. Wasserman K, Hansen JE, Sue DY, et al. Principles of exercise testing and interpretation: including pathophysiology and clinical applications. 5th edn. Baltimore (MD): Lippincott Williams \& Wilkins (LWW), 2011.

28. Balady G, Berra KA, et al. ACSM's Guidelines for exercise testing and prescription. 6th edn. Philadelphia (PA): Lippincott Williams \& Wilkins, 2000.

29. Lohmann TG, Roche AF, Martorell R. Anthropometric standardization reference manual. Champaign (IL): Human Kinetics Books, 1988.

30. American Cancer Society. What are the key statistics about breast cancer? Secondary What are the key statistics about breast cancer? 10 June 2015. http://www.cancer.org/cancer/breastcancer/ detailedguide/breast-cancer-key-statistics (accessed 18 Dec 2015).

31. Schmitz KH, Ahmed RL, Troxel A, et al. Weight lifting in women with breast-cancer-related lymphedema. N Engl J Med 2009;361: 664-73.

32. Schmitz KH, Courneya KS, Matthews C, et al. American College of Sports Medicine roundtable on exercise guidelines for cancer survivors. Med Sci Sports Exerc 2010;42:1409-26.
33. Nutrition Coordinating Center UoM. Nutrition Data System for Research Secondary Nutrition Data System for Research. 2015. http://www.ncc.umn.edu/products/ndsr.html (accessed 18 Dec 2015).

34. Frankenfield D, Roth-Yousey L, Compher C. Comparison of predictive equations for resting metabolic rate in healthy nonobese and obese adults: a systematic review. J Am Diet Assoc 2005;105:775-89.

35. Schenk JM, Neuhouser ML, Lin DW, et al. A dietary intervention to elicit rapid and complex dietary changes for studies investigating the effects of diet on tissues collected during invasive surgical procedures. J Am Diet Assoc 2009;109:459-63.

36. Pi-Sunyer FX, Maggio CA, McCarron DA, et al. Multicenter randomized trial of a comprehensive prepared meal program in type 2 diabetes. Diabetes Care 1999;22:191-7.

37. Rock CL, Doyle C, Demark-Wahnefried W, et al. Nutrition and physical activity guidelines for cancer survivors. CA Cancer J Clin 2012;62:243-74.

38. Kushi LH, Doyle C, McCullough M, et al. American Cancer Society Guidelines on nutrition and physical activity for cancer prevention: reducing the risk of cancer with healthy food choices and physical activity. CA Cancer J Clin 2012;62:30-67.

39. Romaguera D, Vergnaud AC, Peeters $\mathrm{PH}$, et al. Is concordance with World Cancer Research Fund/American Institute for Cancer Research guidelines for cancer prevention related to subsequent risk of cancer? Results from the EPIC study. Am J Clin Nutr 2012;96:150-63.

40. Norat T, Aune D, Chan D, et al. Fruits and vegetables: updating the epidemiologic evidence for the WCRF/AICR lifestyle recommendations for cancer prevention. Cancer Treat Res 2014;159:35-50.

41. Jakicic JM, Clark K, Coleman E, et al. American College of Sports Medicine position stand. Appropriate intervention strategies for weight loss and prevention of weight regain for adults. Med Sci Sports Exerc 2001;33:2145-56.

42. Bandura A. Social foundations of thought and action: a social cognitive theory. Englewood Cliffs (NJ): Prentice-Hall, 1986.

43. Caporaso JG, Lauber CL, Walters WA, et al. Global patterns of 16S rRNA diversity at a depth of millions of sequences per sample. Proc Natl Acad Sci USA 2011;108(Suppl. 1): 4516-22.

44. Daft JG, Ptacek T, Kumar R, et al. Cross-fostering immediately after birth induces a permanent microbiota shift that is shaped by the nursing mother. Microbiome 2015;3:17.

45. Kumar R, Eipers P, Little RB, et al. Getting started with microbiome analysis: sample acquisition to bioinformatics. Curr Protoc Hum Genet 2014:82:18.8.1-18.8.29.

46. Rizza W, Veronese N, Fontana L. What are the roles of calorie restriction and diet quality in promoting healthy longevity? Ageing Res Rev 2014;13:38-45. 